\title{
IMPROVING METHODOLOGICAL STRATEGIES FOR SATELLITE CELLS COUNTING IN HUMAN MUSCLE DURING AGEING
}

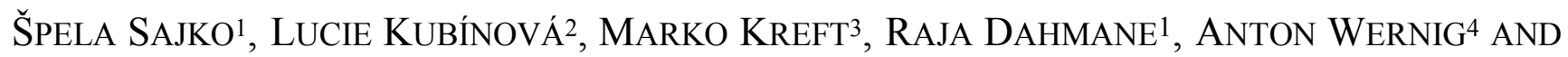 \\ IDA ERŽEN ${ }^{1}$ \\ ${ }^{1}$ Institute of Anatomy, Medical Faculty, 1000 Ljubljana, Slovenia, ${ }^{2}$ Department of Biomathematics, Institute of \\ Physiology, Czech Academy of Sciences, Prague, Czech Republic, ${ }^{3}$ Neuroendocrinology-Molecular Cell \\ Physiology Laboratory, Institute of Pathophysiology, Medical Faculty, 1000 Ljubljana, Slovenia, ${ }^{4}$ Department \\ of Physiology, University Bonn, Bonn, Germany \\ e-mail: spela.sajko@mf.uni-lj.si, ida.erzen@mf.uni-lj.si \\ (Accepted February 15, 2002)
}

\begin{abstract}
Stereological methods, based on the optical disector principle and fluorescent staining, were developed for estimating frequency of satellite cells in skeletal muscles. The parameter $\mathrm{N}_{\mathrm{L}}$ (sc, fib) (number of satellite cells per fibre length) was compared with the parameter $\mathrm{N}_{\mathrm{N}}(\mathrm{sc}$, nucl) (the percentage of satellite cell nuclei in all muscle nuclei), most often published in the literature, by applying unbiased sampling and counting procedures and using a confocal microscope. The methods were tested in autopsy samples of four young vs. four old human vastus lateralis muscles. Both parameters $\mathrm{N}_{\mathrm{L}}\left(\mathrm{sc}\right.$, fib) and $\mathrm{N}_{\mathrm{N}}(\mathrm{sc}$, nucl) declined during ageing. However, it appears that the two parameters cannot be substituted one by the other because the number of nuclei per fibre length tends to be increased during aging. Using the introduced methods, it is more straightforward to estimate $\mathrm{N}_{\mathrm{L}}$ (sc, fib) than $\mathrm{N}_{\mathrm{N}}(\mathrm{sc}$, nucl).
\end{abstract}

Keywords: ageing, immunohistochemistry, optical disector, satellite cells, skeletal muscle.

\section{INTRODUCTION}

Satellite cells are quiescent muscle precursor mononucleated cells situated between basal lamina and plasmalema of adult skeletal muscle fibres. Satellite cells are relatively rare structures, probably evenly distributed along individual muscle fibres, but more concentrated within end plate regions. When activated they enter the mytotic cycle, proliferate and fuse either with each other or with the existing muscle fibres. The main function of a satellite cell is therefore at muscle growth and repair.

There are contradictory reports on whether satellite cell pool is reduced during ageing (Allbrook et al., 1971; Schmalbruch and Hellhammer, 1976; Snow, 1977; Gibson and Schultz, 1983) or not (Hikida et al, 1998; Roth et al. 2000). In most studies, profiles of satellite cell nuclei vs. myonuclei were counted. In general, the methods that have been used to determine satellite cell frequency are mostly biased, the sampling procedure was either not described in sufficient detail, the sample analysed was too small or unproper sampling design was used, therefore the data are not comparable.
The aim of this study was (i) to find a parameter for characterizing the satellite cell frequency in skeletal muscles, i.e. a parameter that is influenced neither by the fibre diameter and volume nor by the contraction state of the muscle and (ii) to measure the parameter most often published in the literature (i.e., the proportion of satellite cells in all myonuclei, $\mathrm{N}_{\mathrm{N}}(\mathrm{sc}$, nucl)), by applying unbiased sampling and design based counting procedures. To test the methods, satellite cell frequency, i.e., $\mathrm{N}_{\mathrm{N}}(\mathrm{sc}$, nucl) and number of satellite cells per fibre length $\mathrm{N}_{\mathrm{L}}$ (sc, fib) were measured in a pilot study of four young vs. old human vastus lateralis muscles.

\section{MATERIALS AND METHODS}

Autopsy samples of vastus lateralis muscle were excised from the border between the middle and the distal part of the femur in two groups of men who suffered sudden death, one young group (aged between 24 and 38 years) and the other old (aged between 67 and 73 years). Four muscles from each group were analysed. Six samples, each about $1 \mathrm{~cm}^{3}$ 
were taken from each muscle: three from the superficial part and three from the deep part and frozen in liquid nitrogen. Using a Reichert-Jung Frigocut 2800, each sample was cut into (section thickness refers to nominal microtome settings only):

(i) three $30 \mu \mathrm{m}$ thick transverse sections with an interval of $100 \mu \mathrm{m}$ to demonstrate basal lamina, satellite cells and nuclei - for satellite cell counting per fibre length,

(ii) one additional $30 \mu \mathrm{m}$ thick transverse section to demonstrate nuclei and basal lamina - for counting all muscle nuclei (i.e. myonuclei + satellite cell nuclei) per fibre length,

(iii) one $10 \mu \mathrm{m}$ thick longitudinal section to demonstrate z-line with desmin - for measuring sarcomere length.

Different sampling schemes in (i) and (ii) were used because the frequency of satellite cells is much lower than that of muscle nuclei, thus it was necessary to evaluate more sections and sampling frames for satellite cell counting.

\section{IMMUNOHISTOCHEMICAL PROCEDURES}

All sections were pretreated in phosphate buffered saline with $0.2 \%$ Triton X-100 (PBS-T) and subsequently fixed in $2 \%$ paraformaldehyde in PBS-T for ten minutes and rinsed in PBS-T. All antibodies were diluted in PBS-T except for M-cadherin (M-cad), which was diluted in $\mathrm{PBS} /$ carrageenan solution (Irintchev et al., 1994).

(i) Satellite cells were demonstrated by double staining of M-cad and laminin. After rinsing in PBS-T, normal goat serum 1:5 was applied for 25 min to reduce background staining. Sections were further incubated overnight in primary rabbit polyclonal antibody against M-cad (1:50) (Institute of Physiology, Bonn) and two hours with goat anti rabbit Alexa Fluor 488 (1:500) (Molecular Probes). After rinsing in PBS-T, basal lamina was detected by incubating overnight with monoclonal antibodies against laminin $(1: 1000)$ (Sigma) followed by two hours with goat anti mouse Alexa-546 antibody (1:500) (Molecular Probes). Bisbenzimide was used to stain nuclei.

Sections were analysed by the two channel Zeiss LSM 510 confocal microscope using the Zeiss Plan-Neofluar oil immersion 40× objective (NA: 1.3), M-cad in green and laminin in red fluorescence. Green and red fluorescence were excited with an argon (488 nm) and $\mathrm{He} / \mathrm{Ne}(543$ $\mathrm{nm})$ laser. Emission signal was filtered using a narrow band (505-530 nm) and a LP $595 \mathrm{~nm}$ filter. The position of nuclei was controlled under the fluorescent microscope (in blue colour).

(ii) On additional sections laminin and myonuclei were demonstrated. After rinsing in PBS-T normal goat serum 1:5 was applied for $25 \mathrm{~min}$. Sections were further incubated overnight with monoclonal anti laminin antibodies (1:1000) (Sigma), and two hours with goat anti mouse Alexa Fluor 488 antibody (1:500). Nuclei were stained with propidium iodide for five minutes. Red coloured myonuclei beneath the green coloured basal lamina were analysed using the above confocal microscope with the $40 \times$ objective.

(iii) Longitudinal sections were incubated with normal rabbit serum 1:5 for $30 \mathrm{~min}$ and overnight with monoclonal anti human desmin $(1: 100)$ (Dako, Denmark) antibody followed by secondary peroxidase conjugated rabbit anti mouse antibodies (1:80) (Dako, Denmark) for one hour. Peroxidase was visualised with diaminobenzidine. The sections were analyzed under a Nikon Eclipse E800 microscope using a planfluor $60 \times$ objective (NA: $0.85)$.

\section{SAMPLING}

(i) Satellite cells: A systematic sampling with a random start was applied to the $30 \mu \mathrm{m}$ thick transverse sections. Samples were taken from every third field of view. Stacks of optical sections, $1 \mu \mathrm{m}$ apart, were captured from a central square field with $x-y$ dimensions of $326 \mu \mathrm{m}$.

(ii) Nuclei (i.e. myonuclei + satellite cell nuclei): As nuclei are very frequent, only six stacks of optical sections, $1 \mu \mathrm{m}$ apart, were captured at random from each $30 \mu \mathrm{m}$ thick section with nuclei stained by propidium iodide.

(iii) Sarcomere length was measured from five randomly chosen muscle fibres from each longitudinal section.

\section{STEREOLOGICAL ANALYSIS}

The following stereological parameters were estimated: number of satellite cells per fibre length $\mathrm{N}_{\mathrm{L}}$ (sc, fib) and number of nuclei (i.e. myonuclei + nuclei of the satellite cells) per fibre length $\mathrm{N}_{\mathrm{L}}$ (nucl, fib). 
To enable comparison of data obtained from specimens frozen at different sarcomere length, both stereological parameters estimated, i.e., number of satellite cells per fibre length $\mathrm{N}_{\mathrm{L}}$ (sc, fib) and number of nuclei per fibre length $\mathrm{N}_{\mathrm{L}}$ (nucl, fib), were corrected to a reference sarcomere length, i.e. divided by the correction factor c. The correction factor $\mathrm{c}$ was calculated by dividing a reference sarcomere length by the measured mean sarcomere length in $\mu \mathrm{m}$. As the reference sarcomere length the value $2.5 \mu \mathrm{m}$ was applied in this study. The value $2.5 \mu \mathrm{m}$ was an average taken from most often indicated length of a resting sarcomere (see Schmalbruch, 1985).

(i) Number of satellite cells per fibre length $\mathrm{N}_{\mathrm{L}}$ (sc, fib): The number of satellite cells $\mathrm{N}(\mathrm{sc})$ was estimated by counting their nuclei applying the optical disector method (Gundersen, 1986) to the sampled stacks (see above), using the DISECTOR software developed by Tomori et al. (2001). This software enables to browse through the stack of confocal images and to superimpose the disector sampling frames on the images. During counting, the individual satellite cell nuclei were marked by a mouse and those sampled by the disector probe were counted. As the distance between the confocal optical sections was $1 \mu \mathrm{m}$, i.e. smaller than any satellite cell nucleus, no stained nucleus could be lost during such measurement. The height of the disector $\mathrm{h}$ was between 10 and 22 $\mu \mathrm{m}$, depending on the penetration of the antibody against laminin. In the disector sampling frames, $319.3 \mu \mathrm{m} \times 319.3 \mu \mathrm{m}$, also the myofibre profiles were counted. It was assumed that the transverse sections are going exactly perpendicular to the muscle fibres, thus the myofibre length L(fib) within the disector probes was equal to the number of myofibre profiles multiplied by the disector height. The appropriate disector height was always used in these calculations. The number of satellite cells per fibre length $\mathrm{N}_{\mathrm{L}}$ (sc, fib) was then estimated by:

$$
e s t N_{L}(s c, f i b)=\frac{N(s c)}{L(f i b)} \cdot \frac{1}{c}
$$

where $\mathrm{N}(\mathrm{sc})$ is the total number of the satellite cell nuclei counted by all disectors and L(fib) is the total length of myofibres within the disector probes and $\mathrm{c}$ is the correction factor for the sarcomere length. On the average, 107 satellite cells (from 36 to 200) within 1352 to 5350 myofibre profiles were counted per muscle.

(ii) Number of nuclei (i.e. myonuclei + nuclei of the satellite cells) per fibre length $\mathrm{N}_{\mathrm{L}}$ (nucl, fib): The number of nuclei $\mathrm{N}$ (nucl) was also estimated by the optical disector method, this time applied to the sampled stacks of $30 \mu \mathrm{m}$ thick sections with nuclei stained by propidium iodide (see above). The disector height was $15 \mu \mathrm{m}$, although the dye penetration was sometimes deeper. In the sampled stacks the depth of the dye penetration was always found to be at least $15 \mu \mathrm{m}$. The total myofibre length L'(fib) within the disector probes (applied to counting nuclei) was calculated in the same way as in (i). The number of nuclei per fibre length $\mathrm{N}_{\mathrm{L}}$ (nucl, fib) was then estimated by:

$$
\operatorname{est}_{L}(n u c l, f i b)=\frac{N(n u c l)}{L^{\prime}(f i b)} \cdot \frac{1}{c}
$$

where $\mathrm{N}$ (nucl) is the total number of nuclei counted by all disectors and $\mathrm{c}$ is the correction factor for the sarcomere length. On the average, 1143 nuclei (from 410 to 1652 ) within 138 to 1167 myofibre profiles were counted per muscle.

(iii) The percentage of satellite cell nuclei $\mathrm{N}_{\mathrm{N}}$ (sc, nucl) in all muscle nuclei was then estimated simply by the ratio of $\mathrm{N}_{\mathrm{L}}$ (sc, fib) to $\mathrm{N}_{\mathrm{L}}$ (nucl, fib) times $100 \%$.

(iv) Sarcomere length was measured at the objective magnification $60 \mathrm{x}$ by counting cross striations per given fibre length. The correction factor $\mathrm{c}$ was calculated by dividing the common reference sarcomere length, i.e. $2.5 \mu \mathrm{m}$ in this study, by the measured mean sarcomere length in $\mu \mathrm{m}$.

\section{STATISTICS}

The differences between the young and the old group were judged by the nonparametric MannWhitney two-sided test.

\section{RESULTS}

Satellite cells were counted by their nuclei, positioned between the basal lamina and M-cad stained satellite cell membranes (Fig. 1a). All those nuclei positioned underneath the basal lamina were counted (Fig. 1b). 

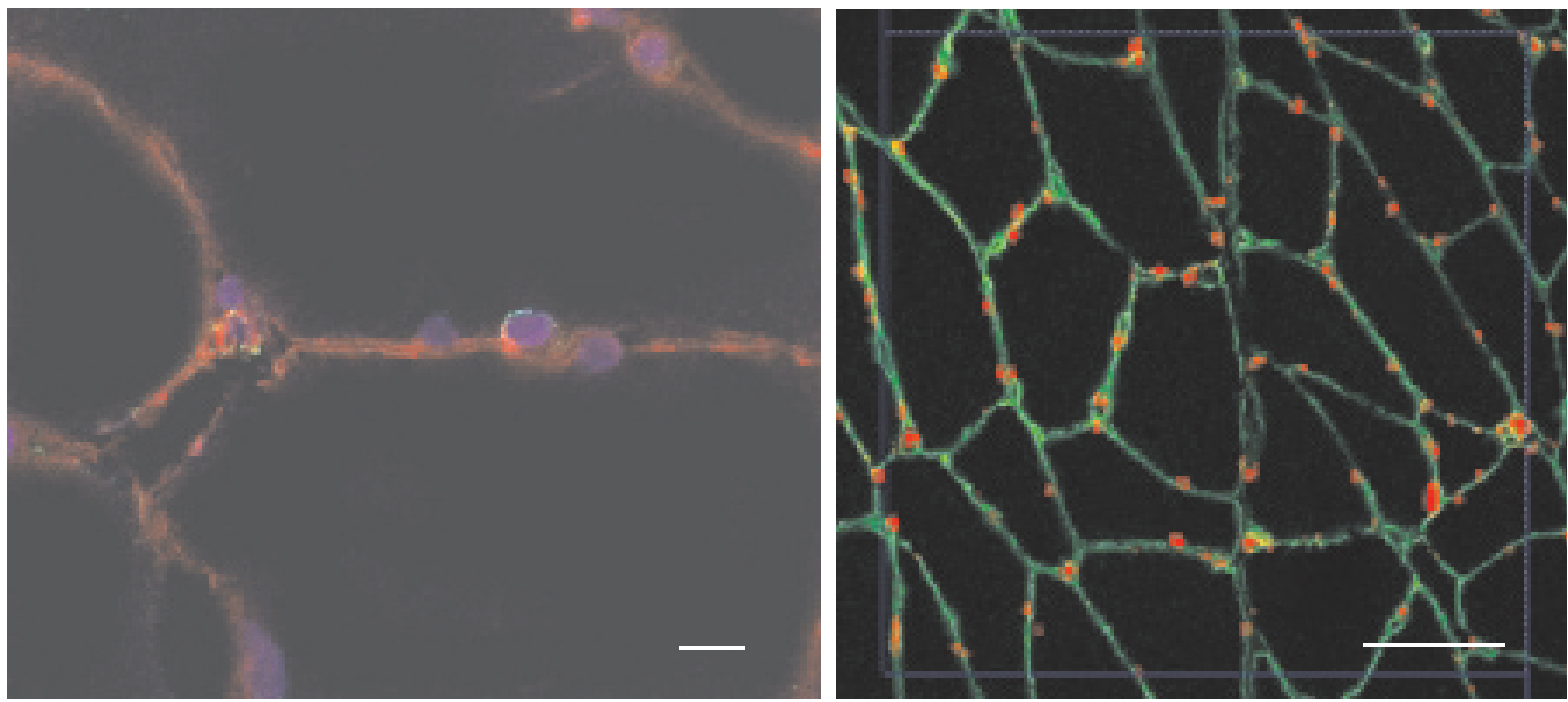

Fig. 1. Skeletal muscle fibres, satellite cell and nuclei: (A) Satellite cell was detected with tripple staining for M-cad (green), laminin (red) and nucleus (blue). Scale bar is 10 um, (B) Basal lamina, stained with laminin (green) and nuclei (red); unbiased counting frame is superimposed. Scale bar is $100 \mu \mathrm{m}$.

Table 1. Frequency of satellite cells in young vs. old human vastus lateralis muscle expressed as number of satellite cells per fibre length $N_{L}(\mathrm{sc}, \mathrm{fib})\left(\mathrm{mm}^{-1}\right)$ and percentage of satellite cell nuclei from total myofibre nuclei $N_{N}(s c, n u c l)(\%)$ and frequency of myonuclei in young vs. old human vastus lateralis muscle expressed as number of nuclei per fibre length $N_{L}(s c, f i b)\left(\mathrm{mm}^{-1}\right)$

\begin{tabular}{|c|c|c|c|c|c|c|c|}
\hline Age & $\mathrm{N}(\mathrm{sc})$ & $\begin{array}{l}\mathrm{L}(\mathrm{fib}) \\
(\mathrm{mm})\end{array}$ & $\begin{array}{c}\mathrm{N}_{\mathrm{L}}(\mathrm{sc}, \text { fib }) \\
(\mathrm{mm}-1)\end{array}$ & $\mathrm{N}$ (nucl) & $\begin{array}{l}\mathrm{L}^{\prime}(\mathrm{fib}) \\
(\mathrm{mm})\end{array}$ & $\begin{array}{c}\mathrm{N}_{\mathrm{L}} \text { (nucl, fib) } \\
(\mathrm{mm}-1)\end{array}$ & $\begin{array}{c}\mathrm{N}_{\mathrm{N}}(\mathrm{sc}, \text { nucl }) \\
(\%)\end{array}$ \\
\hline 24 years & 71 & 26.89 & 1.67 & 583 & 5.27 & 82.09 & 2.03 \\
\hline 28 years & 147 & 37.33 & 2.41 & 410 & 3.23 & 76.58 & 3.15 \\
\hline 30 years & 200 & 86.97 & 1.80 & 1652 & 17.51 & 79.75 & 2.26 \\
\hline 38 years & 153 & 93.22 & 1.34 & 1340 & 10.70 & 103.95 & 1.28 \\
\hline mean & & & 1.81 & & & 85.59 & 2.18 \\
\hline 67 years & 112 & 77.58 & 1.09 & 1617 & 13.59 & 85.08 & 1.28 \\
\hline 69 years & 72 & 50.27 & 1.24 & 1571 & 9.02 & 146.87 & 0.84 \\
\hline 70 years & 65 & 47.45 & 1.16 & 1551 & 7.50 & 160.29 & 0.72 \\
\hline 73 years & 36 & 19.59 & 1.32 & 426 & 2.07 & 151.76 & 0.87 \\
\hline mean & & & 1.20 & & & 136 & 0.93 \\
\hline young:old & & & $\mathrm{p}=0.0286$ & & & $p=0.0571$ & $\mathrm{p}=0.0286$ \\
\hline
\end{tabular}

Sarcomere length was determined by the number of cross striations per fibre length (Fig. 2). Average sarcomere length measured in the samples of eight autopsy muscles ranged from 1.3 to $2.1 \mu \mathrm{m}$.

$\mathrm{N}_{\mathrm{L}}$ (sc, fib) declined during ageing. $\mathrm{N}_{\mathrm{L}}$ (nucl, fib) increased nonsignificantly and consequently $\mathrm{N}_{\mathrm{N}}(\mathrm{sc}$, nucl) decreased (Table 1).

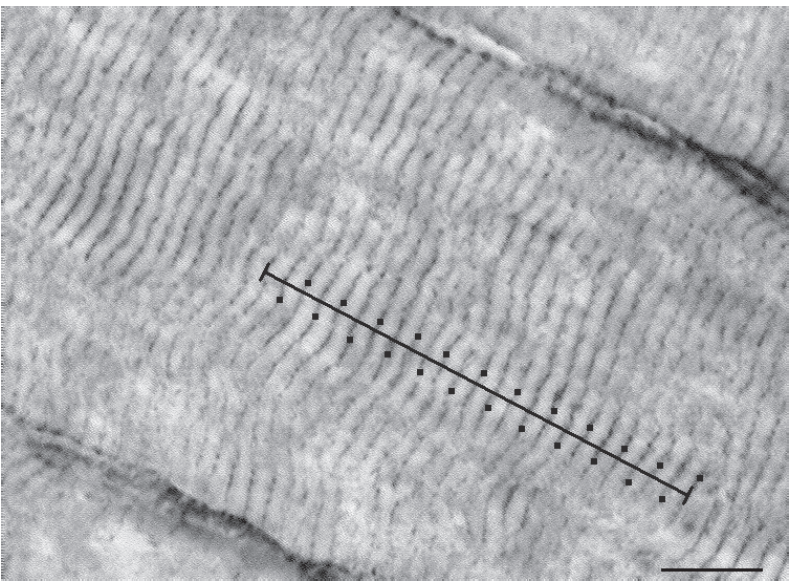

Fig. 2. Sarcomeres were detected by the desmin staining. Scale bar is $10 \mu \mathrm{m}$. 


\section{DISCUSSION}

In this study, satellite cell frequency was compared in young and old human muscles. Using the design based disector principle for counting nuclei and confocal fluorescence microscopy, we tried to overcome some of the deficiencies of previously published methods, i.e., the biased counting profiles of nuclei and satellite cells in single sections (e.g., Hikida et al., 2000) or the timeconsuming and inefficient measurement of the length of nuclei from longitudinal sections at the electron microscopic level (Schmalbruch and Lewis, 2000).

Quantitative evaluation of satellite cell frequency in this study is based on M-cad staining and holds true for M-cad stained satellite cells only. Though specific for mouse satellite cells (Irintchev et al., 1994), the specificity of this marker for human still needs to be proved. From our own experience, high quality tissue preservation is essential for this staining. Moreover, it is not known whether M-cad stains satellite cells in young and old muscles with equal efficiency. As bleaching of the very thin M-cad stained membranes might bias our results, we tried to assure similar exposure of the whole section to the blue light. Therefore, we captured images systematically and only subsequently analyzed the position of the nuclei.

M-cad penetration was deeper than that of laminin, being only $10 \mu \mathrm{m}$ in several cases; in other sections it was much deeper, up to $22 \mu \mathrm{m}$. As the frequency of satellite cells is very low, it was decided to use the largest disector height as possible for the sake of efficiency of the measurement. As a result, the disector height was variable, which was accounted for in the calculations. In the case of counting nuclei (i.e. myonuclei together with satellite cell nuclei), which are more numerous, in all sampled stacks the constant disector height of $15 \mu \mathrm{m}$ was chosen, as the penetration of laminin in these stacks was found to be at least $15 \mu \mathrm{m}$.

In the estimation of $\mathrm{N}_{\mathrm{L}}$ (sc, fib) and $\mathrm{N}_{\mathrm{L}}$ (nucl, fib) we assumed that the transverse sections are going exactly perpendicular to the muscle fibres. Small deviations in the direction of sectioning should not affect the results very much (if the angle $\alpha$ between the cutting direction and the muscle axis is less than $18^{\circ}$, the actual fibre length is by less than $5 \%$ different from the calculated L(fib)). However, if, unlike the present case, the sections were more oblique or if the muscle fibres were not going straight and parallel to the muscle axis, it would be necessary to introduce a simple correction factor (equal to $1 / \cos$ $\alpha$ ). If the fibres are curled the global spatial sampling method (Larsen et al., 1998) which could be applied directly to the stacks of optical sections of the muscle should be used (for practical implementation see the description of the SLICER programme in Kubínová and Janáček, 2001).

Since we were not able to measure sarcomere length from transverse sections we turned the whole tissue sample round and prepared longitudinal sections after we had cut all transverse sections for satellite cell and myonuclei counting. Myofibres are relatively long in human muscles and they run parallel in the samples analysed. Therefore, we asumed that we cut the same fibres that we analysed on transverse section also longitudinally. Autopsy samples of different muscles were practically never frozen at the same sarcomere length. To enable comparison among them, the obtained results were recalculated to a common sarcomere length. The value $2.5 \mu \mathrm{m}$, applied in this study, was an average taken from most often indicated length of a resting sarcomere (see Schmalbruch, 1985). The present study indicates that both parameters $\mathrm{N}_{\mathrm{L}}$ (sc, fib) and $\mathrm{N}_{\mathrm{N}}$ (sc, nucl) decline during ageing. However, we recommend to use $\mathrm{N}_{\mathrm{L}}$ (sc, fib). The two parameters cannot be substituted one by the other as $\mathrm{N}_{\mathrm{L}}$ (nucl, fib) tends to increase during ageing, although the difference was not significant, due to the small number of muscles included into this pilot study. Additionally, $\mathrm{N}_{\mathrm{L}}$ (sc, fib) can be measured faster and can easily be corrected for the contraction state of the muscle recalculating the data to a common reference sarcomere length that should be the same for all muscles compared.

\section{ACKNOWLEDGEMENT}

The study was approved by the National Medical Ethics Committee of Slovenia. Valuable technical assistance of Jerneja Kmecl, Ivan Blažinovič, Marko Slak, Majda Črnak-Maasarani, Andreja Omahen, Katarina Kanc and Milan Števanec is highly acknowledged. The present work was supported by the European programme QLKG-1999-02034; it is a pleasure to acknowledge the most stimulating discussions on methods and results led on several meetings of the consortium.

\section{REFERENCES}

Allbrook DB, Han MF, Helmuth AE (1971). Population of muscle satellite cells in relation to age and mitotic activity. Pathology 3:233-43. 
Gibson MC, Schultz E (1983). Age related differences in absolute numbers of skeletal muscle satellite cells. Muscle Nerve 6:574-80.

Gundersen HJG (1986). Stereology of arbitrary particles. A review of unbiased number and size estimators and the presentation of some new ones, in memory of William R. Thompson. J Microsc 143:3-45.

Hikida RS, Walsh S, Barylski N, Campos G, Hagerman FC, Staron RS (1998). Is hypertrophy limited in elderly muscle fibers? A comparison of elderly and young strength-trained men. BAM 8:419-27.

Irintchev A, Zeschnigk M, Starzinski-Powitz A, Wernig A (1994). Expression pattern of M-cadherin in normal. denervated, and regenerating mouse muscles. Dev Dynamics 199:326-37.

Kubínová L, Janáček J (2001). Confocal microscopy and stereology: estimating volume, number, surface area and length by virtual test probes applied to threedimensional images. Microsc Res Tech 53:425-35.

Larsen JO, Gundersen HJG, Nielsen J (1998) Global spatial sampling with isotropic virtual planes: estimators of length density and total length in thick, arbitrarily orientated sections. J Microsc 191:238-48.

Roth SM, Martel GF, Ivey FM, Lemmer JT, Metter EJ, Hurley BF, Rogers MA (2000). Skeletal muscle satellite cell populations in healthy young and older men and women. Anat Rec 260:351-8.

Schmalbruch H (1985). Skeletal muscle. Berlin, Heidelberg, New York, Toronto: Springer Verlag.

Schmalbruch H, Hellhammer U (1976). The number of satellite cells in normal human muscle. Anat Rec 185:279-88.

Schmalbruch H, Lewis DM (2000). Dynamics of nuclei of muscle fibers and connective tissue cells in normal and denervated rat muscles. Muscle Nerve 23:617-26.

Snow MH (1977). The effects of ageing on satellite cells in skeletal muscles of mice and rats. Cell Tissue Res 185:399-408.

Tomori Z, Krekule I, Kubinova L (2001). DISECTOR program for unbiased estimation of particle number, numerical density and mean volume. Image Anal \& Stereol 20:65-78. 